\title{
The Essentiality of Dynamic Interfaces in Human-Environment Interactions
}

\author{
Manish Arora ${ }^{1^{*}}$, Alessandro Giuliani ${ }^{*}$, Paul Curtin ${ }^{1 *}$ \\ ${ }^{1}$ Department of Environmental Medicine and Public Health, Icahn School of Medicine at \\ Mount Sinai, New York, NY, USA \\ ${ }^{2}$ Environment and Health Department, Istituto Superiore di Sanità, Rome, Italy \\ *authors contributed equally
}

Corresponding Author

Manish Arora

Professor and Vice Chairman

Environmental Medicine and Public Health

Icahn School of Medicine at Mount Sinai

New York, NY 10029

USA

Email: manish.arora@mssm.edu

Subtitle: Complex systems cannot interact directly; they do so through dynamic interfaces

Keywords: biodynamic interface, environment, human, health, conjecture 


\begin{abstract}
The environment impacts human health in profound ways, yet few theories define the form of the relationship between human physiology and the environment. Under current epidemiological constructs of causation, it is assumed that two complex systems (environment and humans) can transfer information directly. This is the underlying structure of the relationship when studies examine, for example, air pollution and brain health. In marked contrast, we conjecture that complex systems cannot interact directly, but rather such interaction requires the formation of an "interface". Further, we contend that this dynamic, process-based interface incorporates components from all the interacting systems but exhibits operational independence. This property has many consequences, the foremost being that characteristics of the interface cannot be fully resolved by only studying the systems involved in the interaction. The interface itself must be the subject of inquiry. Without refocusing our attention on biodynamic interfaces, we cannot discern how our environment impacts health.
\end{abstract}




\section{Introduction}

The interaction between human physiology and the environment is key to deciphering the origins of health and disease. When examining the health consequences of individual agents or mixtures of environmental factors, studies at all layers of organization from human populations to controlled laboratory experiments, are often conceptualized as a mono-directional causative process with the environment impacting some physiologically relevant, measurable end point. In Figure 1a, we depict the general formulation of such a research thesis. Examples include the impact of lead exposure on intelligence quotient (IQ) or social stress exposure and cortisol levels in a biological matrix [1], [2]. Positive environmental influences are also conceptualized in this manner, for example access to green space is associated with better measures of cardiovascular function [3].

More recently, with the realization that the relationship between the environment and human physiology is bi-directional, the arrows visualized in Figure $1 \mathrm{~A}$ can now also point from humans to the environment, as shown in Figure 1B. The scenario depicted in Figure 1B represents a shift from a simple one-directional perturbation to circular feedback causation within and across levels [4], [5]. A simple example of this is the relationship between human industrial activity and air-pollution; our industrial processes increases air-pollution and the increased environmental air-pollution in turn impacts human health [6]. The nature of this relationship has important consequences in how these systems will evolve over time; in a positive feedback cycle, for instance, 
increasing exposures will cause more deleterious health effects, which will in turn lead to increased exposures.

In both Figures 1A and 1B, the often-overlooked aspect is the actual form and physical nature that the interaction between the environment and human physiology can take. Specifically, the underlying assumption in Figures 1A and 1B is that two complex systems (i.e., humans and the environment) can transfer influence directly to one another. In contrast to this, here, we conjecture that complex systems cannot interact directly. We propose that the interaction between two or more complex systems requires the formation of an "interface" (as represented in Figure 1C). Further, we contend that the interface is dynamic process-based in nature (Figure 1D). This interface incorporates components from all the interacting systems but exhibits operational independence. This property has many consequences, the foremost being

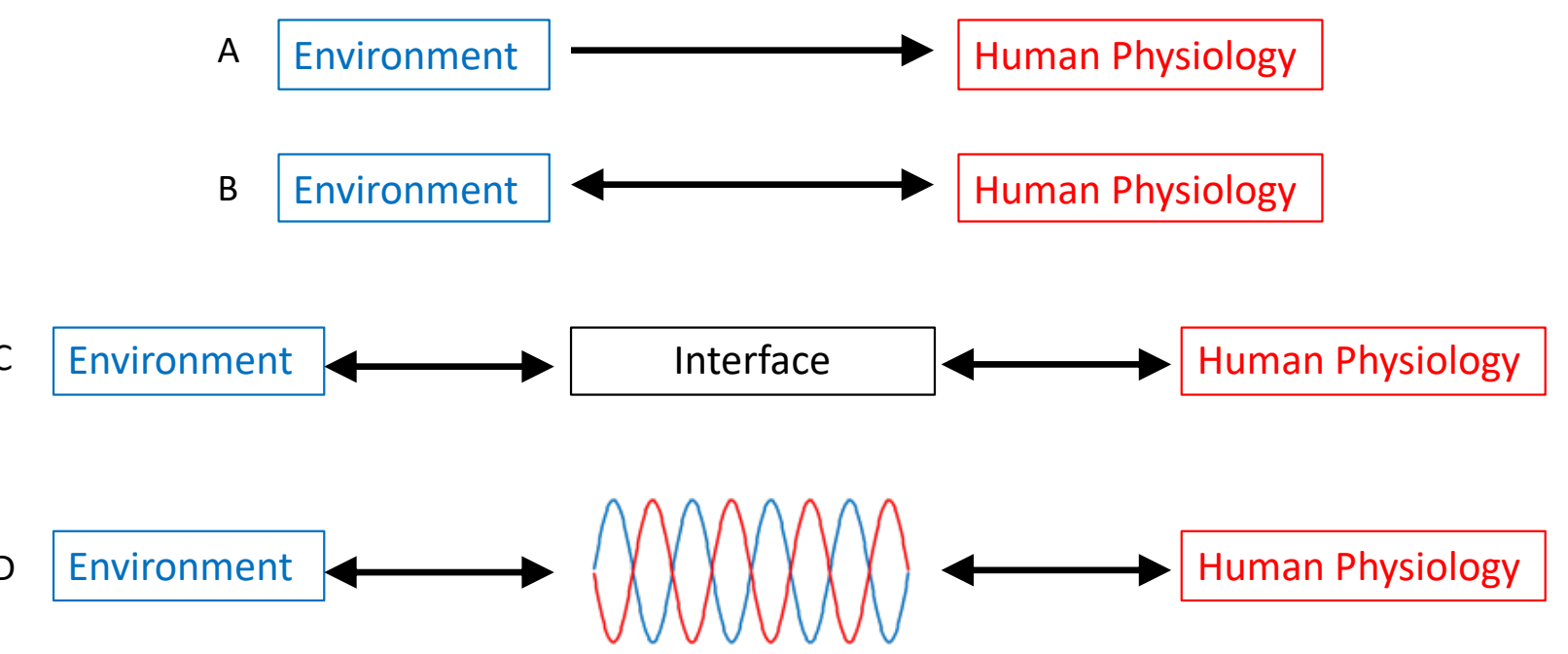

Figure 1. The Biodynamic Interface conjecture. Interactions between the environment and human physiology have been conceptualized as unidirectional (A) and bidirectional (B). We conjecture that complex systems cannot interact directly but do so via one or more interfaces which are composed of components from both the environment and human physiology, but are operationally independent (C). Such interfaces are dynamic (D). 
that the characteristics of the interface cannot be fully resolved by only studying the systems involved in the interaction. The interface must itself be the subject of inquiry. This conjecture would require a shift in the practice of environmental health studies that currently use statistical methods to correlate the variance in one or more environmental measures with the variance in some physiological measures, or some comparable form.

Of importance is the interpretation of direct interaction in the aforementioned conjecture. From a computational perspective, the direct influence of one system on another implies that all the variation in the input/exposure measure relates to the variance in the output/health measure, even if it is partitioned through other measured variables. This is the form that many regression models take when studying the relationship of an environmental exposure on a health outcome and adjust for covariates. In observational studies, researchers are generally and appropriately cautious in interpreting such associations through the lens of a causal analysis, but the ubiquitous application of this framework nonetheless focuses inquiry at the level of reductionist connections between disparate systems. In contrast, we argue that one or more processes contributing to the interface between systems serves to constrain (or in more general terms, assign a "meaning" to) what is transmitted to the other interacting system/s. The biodynamic interface, thus, places limits on both what attributes and how much of the signals are transferred between interacting systems. This happens in both directions; from environment to human, and human to environment. The presence and biological relevance of such intermediary processes may be evident in the time-varying dynamics that link environmental and biological systems, which may not be apparent in direct 
associative studies that seek to link discrete measurements of environmental inputs, often in the form of single measures of concentration, and biological responses.

\section{Evidence for the Existence of Biodynamic Interfaces in Complex System Interactions}

The general premise of what we propose here is pertinent to ideas that exist in other domains of science. In engineering, during the origins of semi-automated control mechanisms between the $17^{\text {th }}$ and $19^{\text {th }}$ centuries, which saw the advent of proportionalintegral-derivative (PID) controllers, the underlying assumptions took a similar form to what we have proposed here [7]. Specifically, the underlying logic of the processes that were developed did not take the form of input $\rightarrow$ control, rather the general form was input $\rightarrow$ state $\rightarrow$ output. Here, the "state" is what decides the transmission of necessary information from the input to the output. Another example is that of a simple mechanical light switch. The action of pressing the button (the input) depends on the current state of the system; if the light is off, then the input would result in it coming on, and vice versa. Although the transmission of the entire variance is an idealized scenario, in a more realistic situation that includes the presence of noise, the constraints imposed by the interface do not blur the underlying correlation but impose a discrete tessellation on the state space into 'permissive' (where the correlation holds) and 'non permissive' (where correlation does not hold) dynamics.

Next, we provide examples from human physiology supporting the conjecture we propose in contexts of basic and clinical research, extending from the level of individuals to molecular interactions to show that dynamic interfaces are not limited to 
any spatial scale or level of observation. At the level of an individual, interfaces emerge in the integration of endogenous organs and systems, and the modulation of these processes by environmental inputs. This is particularly apparent in the interpretation and analysis of human physiology from a clinical perspective. In cardiovascular disease, for example, decades of research has aimed at identifying signatures of healthy and dysregulated function. The cardiac rhythm serves as the final common path for multiple neural systems, including both the endogenous pacemakers which mediate and maintain cardiac rhythm as well as the outputs of the sympathetic and para-sympathetic nervous systems [8], [9]. These so-called "fight or flight" and "rest and digest" systems, respectively, can be elicited in response to external stimuli, for example the reflexive avoidance of a collision; or, in response to our own activity, as more or less strenuous exercise requires necessary resource recruitment. The confluence of internally and externally generated inputs are superimposed on the common path of the cardiac system where the cardiac rhythm acts as an interface comprised of multiple integrated processes. Outside the context of a controlled environment, where various inputs can be isolated, it becomes impossible to determine if a given cardiac rhythm is being driven by the properties of endogenous pacemakers and associated musculature, or by responsiveness to external stimuli. For that reason, assessments of cardiac health are generally conducted under controlled conditions, where a "resting" heart rate can be assessed and contrasted against "stressful" conditions, without intervening activation driven by external inputs [10]. In contrast, in routine non-clinical conditions, where both internally recruited and externally elicited inputs are active, the cardiac rhythm is an interface that constrains the vast amount of information from internal and external 
environment inputs. This essential integration in a relatively well-characterized system would appear elementary to any physiologist; and yet, the essential form of this perspective, which follows the form outlined in Figure 1D, is routinely rejected in epidemiological study designs. Instead the focus of inquiry is a reductionist linkage as illustrated in Figure 1A, sometimes with insertions of molecular intermediates: environmental input $\rightarrow$ molecular marker $\rightarrow$ clinical measure of cardiovascular health.

The constraints on information transfer exerted by dynamic interfaces are also evident in human-to-human interaction. There have been several studies examining the transfer of information between individuals during common tasks that would be encountered in daily routines [11], [12]. For example, in observational studies on individuals participating in unscripted conversations, aspects of language, facial expressions, eye gaze, gestures, posture, and many other attributes may be transferred between the participants. However, what has been repeatedly observed, with different measurement tools, is that while there is clear synchronization in linguistic and physical expression between participants, it only includes a small subset of the measured parameters [11]. In other words, a set of intervening processes constrain what is transferred and synchronized between individuals.

In our own work on molecular networks, we have studied exposure to essential nutrient elements and toxic metals during prenatal and early childhood development and risk of autism spectrum disorder (ASD) diagnosis later in life [13], [14]. In early studies we investigated these relationships through traditional frameworks, essentially akin to Fig. 
$1 \mathrm{~A}$, wherein we created a statistical model to characterize the association between the intensity of metal exposure and the ASD phenotype. From this perspective, we found that the relationship between metals concentrations and ASD phenotype was weak and could not be readily replicated in other populations [13]. More recently, however, we shifted the focus of our studies to examine the dynamics underlying metal assimilation; rather than examine the intensity of exposure alone, we characterized the complexity of cyclical dynamics involved in metal metabolism [14]. Critically, this level of inquiry at the biodynamic interface carries information about both biological and environmental systems; and, as such, possesses emergent properties that may not be evident in either system considered alone. In support of our thesis, of the existence of a dynamic interface, when we considered the biodynamics of elemental assimilation (rhythmicity, for example), we saw consistent and strong associations between elemental exposure and autism phenotype across markedly different populations in three different countries [14]. These examples support that there exists an intermediary process (or set of processes) between environmental exposures and the aspect of human physiology under study, in this case the neurobehavioral phenotype which is disrupted in autism. 
To further illustrate our point, in Figure 2 below, we show a graph based on the wellestablished dynamics of zinc in human blood (zinc dynamics were consistently related to autism phenotype in our studies). The blood levels of zinc reach their peak (of approximately 100 to $110 \mu \mathrm{g} / \mathrm{dL}$ ) in the morning and are lowest in the evening (at round 60 to $70 \mu \mathrm{g} / \mathrm{dL}$ ) [15]. Thus, the interface between environmental (i.e. dietary) zinc exposure and the assimilation of zinc into our biological systems, including the neurological processes impacted in autism spectrum disorder, applies clear constrains on complex environmental inputs during its interaction with human physiology and regulates not only the concentration but also the time-dependent change as it is assimilated into our physiology.

Environmental input
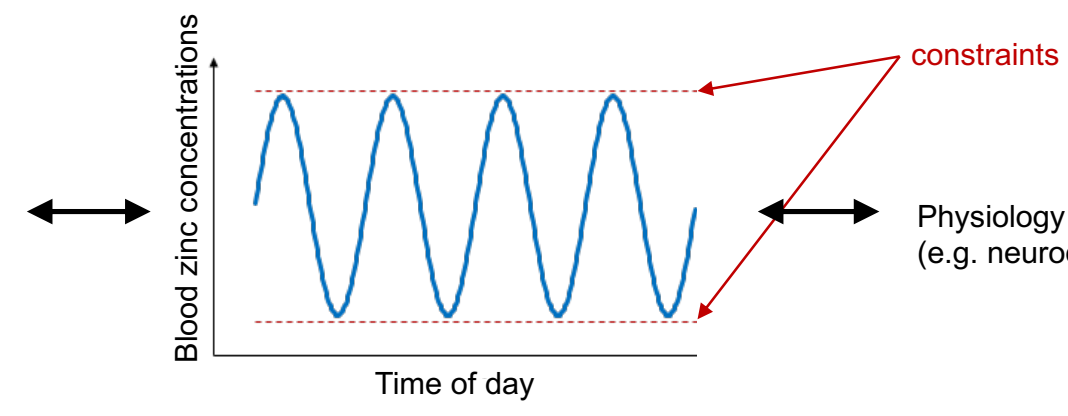

Figure 2. Zinc dynamics over the day as an example of an interface mediating the interaction between environmental inputs (zinc from diet, for example) and physiological systems (neurodevelopment, for example). This biodynamic interface constrains the amount of zinc assimilated from the environment and also how much is made available to the various physiological systems but does so in a time-dependent manner.

The emergence of dynamic interfaces does not appear to be solely a property of highlevel interactions among complex systems, such as social groups, whole organisms, or metabolic networks. On the contrary, even at the levels of organization of two molecules, where the structure and function of proteins drive intracellular organization and intercellular signaling, the ubiquity of dynamic processes is equally evident. As aptly 
stated by Frauenfelder and Wolynes, for example, protein structure is the place where "the physics of complexity and simplicity meet" [16]. Typifying this, while the intermolecular forces involved in protein folding are perfectly known, the capacity to predict the actual fold of a protein molecule remains elusive. Similarly, the functional efficacy of a given protein is not easily deduced from a purely structural model of either the protein itself or the environmental substrate; rather, one needs to understand as well the dynamics that modulate state-dependent functionality.

The functional complexity that emerges from the structural properties of the proteome is clearly evident in the so-called 'allosteric effect'. Allostery is a neologism modeled upon Greek language, which has to do with the ability of proteins to transmit a signal from one site to another in response to environmental stimuli. Of particular relevance to the notion of a dynamic, state-dependent interface, allosteric regulatory mechanisms modulate the efficacy of a given enzyme, either increasing or decreasing the activity associated with a given protein. Although distinct from the effector site at which a given protein directly binds to and acts upon environmental substrates, the activation of allosteric sites can act to alter the functionality of the binding site to modulate the specificity of the enzyme. In this way allosteric modulation provides an essential interface that constrains the functionality of a given protein; one cannot, accordingly, predict the efficacy of a given enzyme according to its structural properties alone, or by its interaction with the environment at its binding site; rather, predicting a given protein's function requires an understanding of its allosteric regulatory state at a given moment. 


\section{Contrasting the Dynamic Interfaces Paradigm from the Current Approaches for Uncovering Mechanisms}

Due to the many advances in technology that have allowed us to study human physiology at ever finer spatial scales of analysis, the focus on mechanism has often taken a reductionist approach, tunneling deeper to discover molecular markers in the expectation that molecules would explain complex phenomenon at all levels of observation, including at societal levels. As a consequence, in studies examining the interaction of the environment and human physiology, the search for mechanisms has taken the form of identifying molecules that may be up- or down-regulated along a pathway diagram. We

provide an analogy here to convey why this approach has had limited success in providing consequential understanding of humanenvironment interactions. In
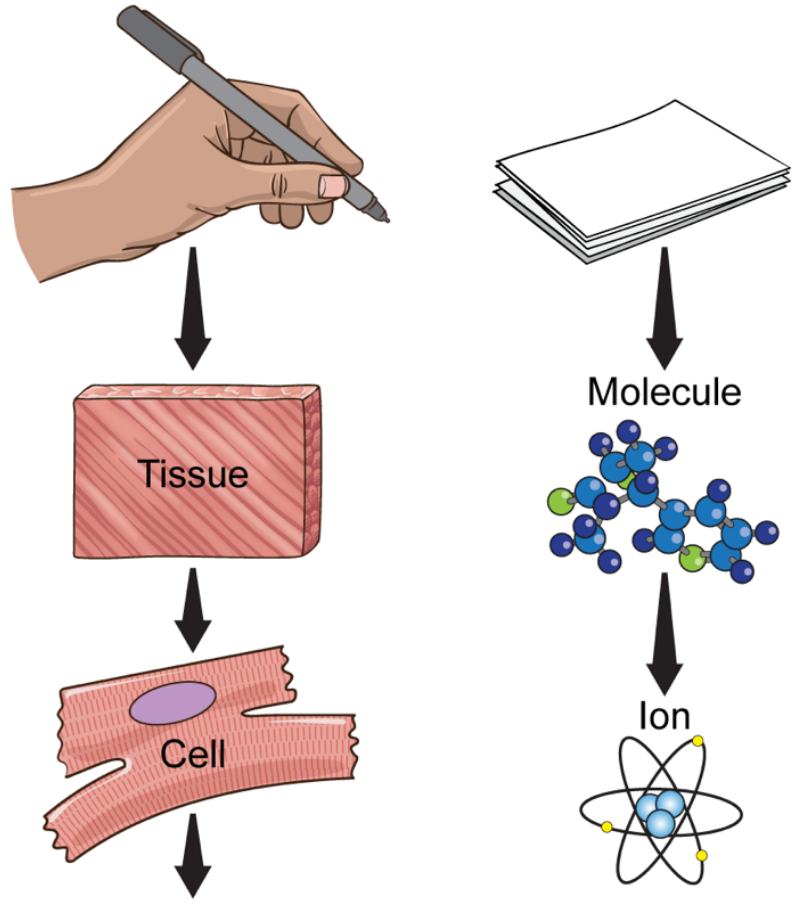
Molecule

Figure 3 we show two

systems interacting—System

$A$ is a human hand that will be transferring information to System B, a piece of paper.

Figure 3. Reductionist approaches that ignore process-based interfaces are unable to uncover key components of system-to-system interactions. Here, analyzing the molecular structure of two systems (hand and paper) cannot reveal the form of the interaction-the handwriting style or the meaning of what is written. Only studying the dynamic interface (the process of writing) can achieve that. 
Under a reductionist approach, System A would be examined in ever smaller spatial scales, from organ to tissue to cellular to the genetic level and, similarly, System B would be examined down to the molecular and ionic levels. However, neither analysis can predict the style of handwriting. To have an accurate measure it is necessary to examine the process-based (and therefore, dynamic) interface that connects the two systems, which is the process of writing. This does not mean that all that is ever written on the piece of paper is necessary. Rather, a small piece of handwriting can be used to deduce the style or pattern of handwriting produced by a person with remarkable detail, as is the case in forensic analysis.

This seemingly mundane detail is important because during any scientific experiment examining system-to-system interactions, the whole set of information is never available but rather the wider set is deduced from a small finite collection of data (i.e. the experimental data).

\section{Implications for Environmental Health Sciences}

If this conjecture holds true, the implication for environmental health sciences are profound. It would shift the current focus from efforts in measuring more and more exposures at lower and lower concentrations to identifying dynamic processes that assimilate exposures into human physiology. It will also require a shift from trying to identify which exposures impact human health to identifying which attributes of the environmental exposures under study are relevant to human health. Alas, we can continue with the current paradigm driven by an genomic era-driven emphasis of digging deeper to smaller spatial scales, and study the finger and the light bulb down to 
their molecular constituents, but we will never establish an accurate relationship between the two unless we examine the state of the interface (whether the system is "on" or "off").

To realize the full potential of the biodynamic interface conjecture, or to prove that it is incorrect, environmental health science must refocus the examination of the interaction of environment and health from an emphasis on measuring physiological "moments", (i.e. static measures of environmental factors, infrequent anthropometry, momentary health indicators) to studying dynamic human-environment interfaces, physiologic states and the processes that constrain to those states. To this end, we provide a set of endeavors that must be undertaken to capitalize on the biodynamic interface paradigm:

1. To focus our scientific inquiry on interfaces which connect biological and environmental systems. An important consequence of the conjecture we propose is that studying the input and output systems will not permit complete characterization of the interface. The interface is not a derivative of either system and must be studied directly. Given that the interface may exhibit complexity independent of the systems contributing to its emergence, we must focus on the characteristic emergent complexity, self-organization, state-dependency, and sensitivity to initial conditions.

2. To develop theoretical frameworks that focus on the identification and interpretation of constraints in biological-environmental interfaces. The constraints acting upon a given interface will ultimately determine the organization of the interface, its 
response to perturbation and subsequently the phenotypic 'output' signal. Analyzing correlations between different measures of the environment and human physiology without characterizing the constrains will not yield a fully explanatory model.

3. To develop laboratory, clinical, and epidemiological methods to relate the complexity characterized at the level of biodynamic interfaces to human health, particularly with regard to the interfaces of processes that unite humans and their environments. We need to measure processes with better specificity in the dimensions of space and time. Explicitly, this requires the rejection of epidemiologic study designs that ignore processes and measure the environment and human physiology as static entities. At a conceptual level, we must reject a purely structural reductionist perspective.

\section{Conclusion}

We are taught that an atom is made of electrons moving around a nucleus containing protons and neutrons. What is overlooked is that one of the most important components of an atom is the seemingly empty space between the electrons and the nucleus. Without this space, matter as we know it would cease to exist. The importance of that space is not that it separates the atom's electrons from its nucleus, but that it is where so much happens-that space is where electrons interact with the nucleus of their own atom. When an atom is perturbed (by the ingress of energy in the form of $X$ rays during fluorescence, for example), it is this space where electrons rearrange themselves to achieve a stable configuration. It is also this seemingly empty space where electrons from one atom interact with those of other atoms, and thus chemical reactions, including those that gave rise to life, begin. The same is true at much larger spatial scales- 
between all the planets, moons and stars and other heavenly bodies, there is the essential space that facilitates gravity, radiation, and the evolution of our physical universe. Why should it be any different for human-environment interactions?

It is also pertinent that we place our thesis in context of the important work of others that reject homeostasis in favor of homeodynamics [17], that extend relativistic concepts from physics to biology [4], [5], that accept the ever-changing nature of our biology and argue for the existence of critical windows of susceptibility [18], and those that study human biology as a network of systems [19]. Many of these scientific theories and methods rely on the existence of levels, scales and states defined by their properties, and compartments defined by their structures and/or functions. Because of this, the direction of interaction across levels has been, and rightly so, the focus of much scientific debate [4], [5]. These levels, scales and compartments are necessary, but let us not forget that equally necessary is what exists between them. Here, we have turned our gaze to this seemingly empty and undefined space, and in doing so, we have presented an argument, not about the direction of interaction or causality between systems and their components, but where the interaction between systems exists, and what makes causal linkages possible—dynamic interfaces. 


\section{Conflict of Interest}

The authors declare no conflict of interest.

\section{Acknowledgment}

MA is funded by National Institute of Environmental Health Sciences grants P30ES023515, DP2ES025453, R01ES026033, U2CES030859. PC is funded by National Institute of Environmental Health Sciences grants U2CES026555.

\section{References}

1 Lanphear BP, Hornung R, Khoury J, Yolton K, et al. 2005. Low-level environmental lead exposure and children's intellectual function: an international pooled analysis. Environ Health Perspect 113: 894-9.

2 Bunea IM, Szentagotai-Tatar A, Miu AC. 2017. Early-life adversity and cortisol response to social stress: a meta-analysis. Transl Psychiatry 7: 1274.

3 Seo S, Choi S, Kim K, Kim SM, et al. 2019. Association between urban green space and the risk of cardiovascular disease: A longitudinal study in seven Korean metropolitan areas. Environ Int 125: 51-7.

4 Noble R, Tasaki K, Noble PJ, Noble D. 2019. Biological Relativity Requires Circular Causality but Not Symmetry of Causation: So, Where, What and When Are the Boundaries? Front Physiol 10: 827.

5 Noble D. 2012. A theory of biological relativity: no privileged level of causation. Interface Focus 2: 55-64.

6 Grandjean P, Landrigan PJ. 2006. Developmental neurotoxicity of industrial chemicals. Lancet 368: 2167-78.

7 Bellman RE. 2016. Adaptive Control Processes A Guided Tour.

8 Nerbonne JM, Kass RS. 2005. Molecular physiology of cardiac repolarization. Physiol Rev 85: 1205-53.

9 Klabunde RE. 2017. Cardiac electrophysiology: normal and ischemic ionic currents and the ECG. Adv Physiol Educ 41: 29-37.

10 Adachi H. 2017. Cardiopulmonary Exercise Test. Int Heart J 58: 654-65.

11 Louwerse MM, Dale R, Bard EG, Jeuniaux P. 2012. Behavior matching in multimodal communication is synchronized. Cogn Sci 36: 1404-26.

12 Kawasaki M, Yamada Y, Ushiku Y, Miyauchi E, et al. 2013. Inter-brain synchronization during coordination of speech rhythm in human-to-human social interaction. Sci Rep 3: 1692.

13 Arora M, Reichenberg A, Willfors C, Austin C, et al. 2017. Fetal and postnatal metal dysregulation in autism. Nat Commun 8: 15493. 
14 Curtin P, Austin C, Curtin A, Gennings C, et al. 2018. Dynamical features in fetal and postnatal zinc-copper metabolic cycles predict the emergence of autism spectrum disorder. Sci Adv 4: eaat1293.

15 Scales WE, Vander AJ, Brown MB, Kluger MJ. 1988. Human circadian rhythms in temperature, trace metals, and blood variables. J Appl Physiol (1985) 65: 1840-6.

16 Frauenfelder H, Wolynes PG. 1994. Biomolecules: Where the Physics of Complexity and Simplicity Meet. PHYSICS TODAY 47: 58.

17 Lloyd D, Aon MA, Cortassa S. 2001. Why homeodynamics, not homeostasis? ScientificWorldJournal 1: 133-45.

18 Selevan SG, Kimmel CA, Mendola P. 2000. Identifying critical windows of exposure for children's health. Environ Health Perspect 108 Suppl 3: 451-5.

19 Ma'ayan A. 2017. Complex systems biology. J R Soc Interface 14. 